\title{
Inertial Iteration for Split Common Fixed-Point Problem for Quasi-Nonexpansive Operators
}

\author{
Yazheng Dang ${ }^{1,2}$ and Yan Gao ${ }^{1}$ \\ ${ }^{1}$ School of Management, University of Shanghai for Science and Technology, Shanghai 200093, China \\ ${ }^{2}$ College of Computer Science and Technology, Henan Polytechnic University, Jiaozuo 454000, China \\ Correspondence should be addressed to Yazheng Dang; jgdyz@163.com
}

Received 14 March 2013; Accepted 6 May 2013

Academic Editor: Ru Dong Chen

Copyright (c) 2013 Y. Dang and Y. Gao. This is an open access article distributed under the Creative Commons Attribution License, which permits unrestricted use, distribution, and reproduction in any medium, provided the original work is properly cited.

\begin{abstract}
Inspired by the note on split common fixed-point problem for quasi-nonexpansive operators presented by Moudafi (2011), based on the very recent work by Dang et al. (2012), in this paper, we propose an inertial iterative algorithm for solving the split common fixed-point problem for quasi-nonexpansive operators in the Hilbert space. We also prove the asymptotical convergence of the algorithm under some suitable conditions. The results improve and develop previously discussed feasibility problems and related algorithms.
\end{abstract}

\section{Introduction}

The convex feasibility problem (CFP), as an important optimization problem [1], is to find a common point in the intersection of finitely many convex sets. It has been applied to many areas, for instance, approximation theory [2], image reconstruction from projections [3, 4], control [5], and so on. When there are only two sets and constraints are imposed on the solutions in the domain of a linear operator as well as in this operator's ranges, the problem is said to be a split feasibility problem (SFP) which has the following formula: finding a point $x$ satisfying

$$
x \in C, \quad A x \in Q \text {, }
$$

where $C$ is a closed convex subset of a Hilbert space $H_{1}, Q$ is a closed convex subset of a Hilbert space $H_{2}$, and $A$ : $H_{1} \rightarrow H_{2}$ is a bounded linear operator. The SFP was originally introduced in [6], and it has also broad applications in many fields, such as image reconstruction problem, signal processing, and radiation therapy. Many projection methods have also been developed for solving the SFP; see [7-9]. Denote by $P_{C}$ the orthogonal projection onto $C$; that is, $P_{C}(x)=\arg \min _{y \in C}\|x-y\|$, over all $x \in C$. Assuming that the SFP is consistent (i.e., (1) has a solution), it is not hard to see that $x \in C$ solves (1) if and only if it solves the fixed-point equation:

$$
x=P_{C}\left[\left(I-\gamma A^{*}\left(I-P_{\mathrm{Q}}\right) A\right)(x)\right],
$$

where $0<\gamma$ is any positive constant and $A^{*}$ denotes the adjoint of $A$.

To solve (2), in [10], Byrne introduced the so-called CQ algorithm, which generates a sequence $\left\{x^{k}\right\}$ by

$$
x^{k+1}=P_{C}\left[\left(I-\gamma A^{*}\left(I-P_{\mathrm{Q}}\right) A\right)\left(x^{k}\right)\right],
$$

where $0<\gamma<2 / \rho\left(A^{T} A\right)$ and $\rho\left(A^{T} A\right)$ is the spectral radius of $A^{*} A$.

The split common fixed-point problem (SCFP) is a generalization of the split feasibility problem (SFP) and the convex feasibility problem (CFP); see [11]. Our main purpose here is to give an extension of the results developed in [12] to the split common fixed-point problem for quasi-nonexpansive operators, and we will introduce weak symposium convergence 
result of the algorithm under some suitable conditions. This will be done in the context of general Hilbert spaces.

The paper is organized as follows. In Section 2, we recall some preliminaries. In Section 3, we present an inertial CQ algorithm and show its convergence.

\section{Preliminaries}

Throughout the rest of the paper, $I$ denotes the identity operator and $\operatorname{Fix}(T)$ denotes the set of the fixed points of an operator $T$, that is, $\operatorname{Fix}(T):=\{x \mid x=T(x)\}$. $\left(\varepsilon_{\mathrm{Q}}\right)$ if

Recall that a mapping $T$ is said to be quasi-nonexpansive

$$
\|T x-q\| \leq\|x-q\|, \quad \forall(x, q) \in H \times \operatorname{Fix}(T) .
$$

A mapping $T$ is called nonexpansive $\left(\varepsilon_{\mathrm{N}}\right)$ if

$$
\|T(x)-T(y)\| \leq\|x-y\|, \quad \forall(x, y) \in H \times H .
$$

A mapping $T$ is called firmly nonexpansive $\left(\varepsilon_{\mathrm{FN}}\right)$ if

$$
\begin{array}{r}
\|T(x)-T(y)\|^{2} \leq\|x-y\|^{2}-\|(x-y)-(T(x)-T(y))\|^{2}, \\
\forall(x, y) \in H \times H .
\end{array}
$$

A mapping $T$ is called firmly quasi-nonexpansive $\left(\varepsilon_{\mathrm{FQ}}\right)$ if

$$
\begin{array}{r}
\|T(x)-q\|^{2} \leq\|x-q\|^{2}-\|x-T(x)\|^{2}, \\
\forall(x, q) \in H \times \operatorname{Fix}(H) .
\end{array}
$$

It is easily observed that $\varepsilon_{\mathrm{FN}} \subset \varepsilon_{\mathrm{N}} \subset \varepsilon_{\mathrm{Q}}$ and that $\varepsilon_{\mathrm{FN}} \subset \varepsilon_{\mathrm{FQ}} \subset$ $\varepsilon_{\mathrm{Q}}$. Furthermore, $\varepsilon_{\mathrm{FN}}$ is well known to include resolvents and projection operators, while $\varepsilon_{\mathrm{FQ}}$ contains subgradient projection operators (see, e.g., [13], and the references therein).

Recently, Bauschke and Combettes [14] have considered a class of mappings satisfying the condition

$$
\langle q-T x, x-T x\rangle \leq 0, \quad \forall(x, q) \in H \times \operatorname{Fix}(T) .
$$

It can easily be seen that the class of mappings satisfying the latter condition coincides with that of firmly quasinonexpansive mappings.

Usually, the convergence of fixed-point algorithms requires some additional smoothness properties of the mapping $T$ such as demiclosedness.

Definition 1. A mapping $T$ is said to be demiclosed if for any sequence $\left\{x^{k}\right\}$ which weakly converges to $y$ and if the sequence $\left\{T\left(x^{k}\right)\right\}$ strongly converges to $z$, then $T(y)=z$.

In what follows, only the particular case of demiclosedness at zero will be used, which is the particular case when $z=0$.

The following lemmas will be needed in the proof of the convergence of the algorithm.
Lemma 2. Let $T$ be a quasi-nonexpansive mapping. Set $T_{\alpha}:=$ $(1-\alpha) I+\alpha T$. Then, it is immediate that for all $(x, q) \in H \times$ $\operatorname{Fix}(T)$ :

$$
\begin{aligned}
& \text { (1) }\langle x-T(x), x-q\rangle \geq(1 / 2)\|x-T(x)\|^{2} \text { and }\langle x-T(x), q- \\
& \quad T(x)\rangle \leq(1 / 2)\|x-T(x)\|^{2} \text {; } \\
& \text { (2) }\left\|T_{\alpha}(x)-q\right\|^{2} \leq\|x-q\|^{2}-\alpha(1-\alpha)\|x-T(x)\| \text {; } \\
& \text { (3) }\left\langle x-T_{\alpha}(x), x-q\right\rangle \geq(\alpha / 2)\|x-T(x)\|^{2} \text {. }
\end{aligned}
$$

Lemma 3 (see $[8]$ ). Assume $\varphi_{k} \in[0, \infty)$ and $\delta_{k} \in[0, \infty)$ satisfy

(1) $\varphi_{k+1}-\varphi_{k} \leq \theta_{k}\left(\varphi_{k}-\varphi_{k-1}\right)+\delta_{k}$,

(2) $\sum_{k=1}^{+\infty} \delta_{k}<\infty$,

(3) $\left\{\theta_{k}\right\} \subset[0, \theta]$, where $\theta \in[0,1)$.

Then, the sequence $\left\{\varphi_{k}\right\}$ is convergent with $\sum_{k=1}^{+\infty}\left[\varphi_{k+1}-\varphi_{k}\right]_{+}<$ $\infty$, where $[t]_{+}:=\max \{t, 0\}$ (for any $t \in R$ ).

\section{The Inertial Algorithm and Its Asymptotic Convergence}

In what follows, we will focus our attention on the following general two-operator split common fixed-point problem:

$$
\text { find } x^{*} \in C \text { such that } A x^{*} \in Q \text {, }
$$

where $A: H_{1} \rightarrow H_{2}$ is a bounded linear operator and $U$ : $H_{1} \rightarrow H_{1}$ and $T: H_{2} \rightarrow H_{2}$ are two quasi-nonexpansive operators with nonempty fixed-point sets $\operatorname{Fix}(U)=C$ and $\operatorname{Fix}(T)=Q$, and denote the solution set of the two-operator SCFP by

$$
\Gamma=\{y \in C: A y \in Q\}
$$

3.1. The Inertial Algorithm. To solve (9), Moudafi [15] proposed and proved, in finite-dimensional spaces, the convergence of the following algorithm:

$$
x^{k+1}=U_{\alpha_{k}}\left(x^{k}+\gamma\left(A^{*} T_{\beta}-I\right) A\left(x^{k}\right)\right), \quad k \in N,
$$

where $\beta \in(0,1), \alpha_{k} \in(0,1)$ are relaxation parameters and $\gamma>0$. Inspired by the inertial technique, we introduce the following inertial method and then present its convergence analysis.

\section{Algorithm 4.}

Initialization: Let $x^{0} \in H^{1}$ be arbitrary.

Iterative step: For $k \in N$, set $u=I+\gamma \eta A^{*}(T-I) A$, and let

$$
\begin{gathered}
y^{k}=x^{k}+\theta_{k}\left(x^{k}-x^{k-1}\right) \\
x^{k+1}=\left(1-\alpha_{k}\right) u\left(y^{k}\right)+\alpha_{k} U\left(u\left(y^{k}\right)\right), \quad k \in N,
\end{gathered}
$$


where $\eta \in(0,1), \alpha_{k} \in(0,1)$, and $\gamma \in(0,1 /(\lambda \eta))$, with $\lambda$ being the spectral radius of the operator $A^{*} A, \theta_{k} \in[0,1)$.

3.2. Asymptotic Convergence of the Inertial Algorithm. In this subsection, we establish the asymptotic convergence of Algorithm 4.

Lemma 5 (Opial [16]). Let $H$ be a Hilbert space and let $\left\{x^{k}\right\}$ be a sequence in $H$ such that there exists a nonempty set $S \subset H$ satisfying

(1) for every $x^{*}, \lim _{k}\left\|x^{k}-x^{*}\right\|$ exists,

(2) any weak cluster point of the sequence $\left\{x^{k}\right\}$ belongs to $S$. Then, there exists $z \in S$ such that $\left\{x^{k}\right\}$ weakly converges to $z$.

Theorem 6. Given a bounded linear operator $A: H_{1} \rightarrow H_{2}$, let $U: H_{1} \rightarrow H_{1}$ be a quasi-nonexpansive operator with nonempty $\operatorname{Fix}(U)=\mathrm{C}$ and let $\mathrm{T}: \mathrm{H}_{2} \rightarrow \mathrm{H}_{2}$ be a quasinonexpansive operator with nonempty $\operatorname{Fix}(T)=Q$. Assume that $U-I$ and $T-I$ are demiclosed at 0 . If $\Gamma \neq \emptyset$, then any sequence $\left\{x^{k}\right\}$ generated by Algorithm 4 weakly converges to a split common fixed point, provided that we choose $\theta_{k}$ satisfying $\theta_{k} \in\left[0, \bar{\theta}_{k}\right]$ with $\bar{\theta}_{k}:=\min \left\{\theta, 1 /\left(k\left\|x^{k}-x^{k-1}\right\|\right)^{2}\right\}, \theta \in[0,1)$. $\gamma \in(0,1 /(\lambda \eta))$ and $\alpha_{k} \in(\delta, 1-\delta)$ for a small enough $\delta>0$.

Proof. Taking $z \in \Gamma$, and using (2) in Lemma 2, we obtain

$$
\begin{aligned}
& \left\|x^{k+1}-z\right\|^{2} \\
& \quad=\left\|\left(1-\alpha_{k}\right) u\left(y^{k}\right)+\alpha_{k} U\left(u\left(y^{k}\right)\right)-z\right\|^{2} \\
& \quad \leq\left\|u\left(y^{k}\right)-z\right\|^{2}-\alpha_{k}\left(1-\alpha_{k}\right)\left\|U\left(u\left(y^{k}\right)\right)-u\left(y^{k}\right)\right\|^{2} .
\end{aligned}
$$

On the other hand, we have

$$
\begin{aligned}
\left\|u\left(y^{k}\right)-z\right\|^{2}= & \left\|y^{k}+\gamma \eta A^{*}(T-I)\left(A y^{k}\right)-z\right\|^{2} \\
= & \left\|y^{k}-z\right\|^{2}+\gamma^{2} \eta^{2}\left\|A^{*}(T-I)\left(A y^{k}\right)\right\|^{2} \\
& +2 \gamma \eta\left\langle y^{k}-z, A^{*}(T-I)\left(A y^{k}\right)\right\rangle \\
\leq & \left\|y^{k}-z\right\|^{2}+\lambda \gamma^{2} \eta^{2}\left\|(T-I)\left(A y^{k}\right)\right\|^{2} \\
& +2 \gamma \eta\left\langle A y^{k}-A z,(T-I)\left(A y^{k}\right)\right\rangle,
\end{aligned}
$$

that is,

$$
\begin{aligned}
\left\|u\left(y^{k}\right)-z\right\|^{2} \leq & \left\|y^{k}-z\right\|^{2}+\lambda \gamma^{2} \eta^{2}\left\|(T-I)\left(A y^{k}\right)\right\|^{2} \\
& +2 \gamma \eta\left\langle A y^{k}-A z,(T-I)\left(A y^{k}\right)\right\rangle .
\end{aligned}
$$

Now, by setting $v:=2 \gamma \eta\left\langle A y^{k}-A z,(T-I)\left(A y^{k}\right)\right\rangle$ and using (1) of Lemma 2, we obtain

$$
\begin{aligned}
v= & 2 \gamma \eta\left\langle A y^{k}-A z,(T-I)\left(A y^{k}\right)\right\rangle \\
= & 2 \gamma \eta\left\langle A y^{k}-A z+(T-I)\left(A y^{k}\right)\right. \\
& \left.-(T-I)\left(A y^{k}\right),(T-I)\left(A y^{k}\right)\right\rangle \\
= & 2 \gamma \eta\left(\left\langle T\left(A y^{k}\right)-A z,(T-I)\left(A y^{k}\right)\right\rangle\right. \\
& \left.-\left\|(T-I)\left(A y^{k}\right)\right\|^{2}\right) \\
\leq & 2 \gamma \eta\left(\frac{1}{2}\left\|(T-I)\left(A y^{k}\right)\right\|^{2}-\left\|(T-I)\left(A y^{k}\right)\right\|^{2}\right) \\
\leq & -\gamma \eta\left\|(T-I)\left(A y^{k}\right)\right\|^{2} .
\end{aligned}
$$

Combining the key inequality above with (15) yields

$$
\begin{aligned}
\left\|x^{k+1}-z\right\|^{2} \leq & \left\|y^{k}-z\right\|^{2} \\
& -\gamma \eta(1-\lambda \gamma \eta)\left\|(T-I)\left(A y^{k}\right)\right\|^{2} \\
& -\alpha_{k}\left(1-\alpha_{k}\right)\left\|U\left(u\left(y^{k}\right)\right)-u\left(y^{k}\right)\right\|^{2} .
\end{aligned}
$$

Define the auxiliary real sequence $\varphi_{k}:=(1 / 2)\left\|x^{k}-z\right\|^{2}$. Therefore, from (17), we have

$$
\begin{aligned}
\varphi_{k+1} \leq & \frac{1}{2}\left\|y^{k}-z\right\|^{2} \\
& -\frac{1}{2} \gamma \eta(1-\lambda \gamma \eta)\left\|(T-I)\left(A y^{k}\right)\right\|^{2} \\
& -\frac{1}{2} \alpha_{k}\left(1-\alpha_{k}\right)\left\|U\left(u\left(y^{k}\right)\right)-u\left(y^{k}\right)\right\|^{2} .
\end{aligned}
$$

By deducing, we have

$$
\begin{aligned}
\frac{1}{2}\left\|y^{k}-z\right\|^{2}= & \frac{1}{2}\left\|x^{k}+\theta_{k}\left(x^{k}-x^{k-1}\right)-z\right\|^{2} \\
= & \frac{1}{2}\left\|x^{k}-z\right\|^{2}+\theta_{k}\left\langle x^{k}-z, x^{k}-x^{k-1}\right\rangle \\
& +\frac{\theta_{k}^{2}}{2}\left\|x^{k}-x^{k-1}\right\|^{2} \\
= & \varphi_{k}+\theta_{k}\left\langle x^{k}-z, x^{k}-x^{k-1}\right\rangle \\
& +\frac{\theta_{k}^{2}}{2}\left\|x^{k}-x^{k-1}\right\|^{2} .
\end{aligned}
$$

It is easy to check that $\varphi_{k}=\varphi_{k-1}+\left\langle x^{k}-z, x^{k}-x^{k-1}\right\rangle-$ $(1 / 2)\left\|x^{k}-x^{k-1}\right\|^{2}$.

Hence,

$$
\begin{aligned}
\frac{1}{2}\left\|y^{k}-z\right\|^{2}= & \varphi_{k}+\theta_{k}\left(\varphi_{k}-\varphi_{k-1}\right) \\
& +\frac{\theta_{k}+\theta_{k}^{2}}{2}\left\|x^{k}-x^{k-1}\right\|^{2} .
\end{aligned}
$$


Putting (20) into (18), we get

$$
\begin{aligned}
\varphi_{k+1} \leq & \varphi_{k}+\theta_{k}\left(\varphi_{k}-\varphi_{k-1}\right) \\
& +\frac{\theta_{k}+\theta_{k}^{2}}{2}\left\|x^{k}-x^{k-1}\right\|^{2} \\
& -\frac{1}{2} \gamma \eta(1-\lambda \gamma \eta)\left\|(T-I)\left(A y^{k}\right)\right\|^{2} \\
& -\frac{1}{2} \alpha_{k}\left(1-\alpha_{k}\right)\left\|U\left(u\left(y^{k}\right)\right)-u\left(y^{k}\right)\right\|^{2} .
\end{aligned}
$$

Since $\gamma \in(0,1 /(\lambda \eta))$, according to $\theta_{k}^{2} \leq \theta_{k}, \alpha_{k} \in(0,1)$ and (21), we derive

$$
\varphi_{k+1} \leq \varphi_{k}+\theta_{k}\left(\varphi_{k}-\varphi_{k-1}\right)+\theta_{k}\left\|x^{k}-x^{k-1}\right\|^{2} .
$$

Evidently,

$$
\sum_{k=1}^{+\infty} \theta_{k}\left\|x^{k}-x^{k-1}\right\|^{2}<\infty,
$$

due to $\theta_{k}\left\|x^{k}-x^{k-1}\right\|^{2} \leq 1 / k^{2}$. Let $\delta_{k}:=\theta_{k}\left\|x^{k}-x^{k-1}\right\|^{2}$ in Lemma 3. We deduce that the sequence $\left\{\left\|x^{k}-z\right\|\right\}$ is convergent (hence, $\left\{x^{k}\right\}$ is bounded). By (23) and Lemma 3, we obtain $\sum_{k=1}^{+\infty}\left[\left\|x^{k}-z\right\|^{2}-\left\|x^{k-1}-z\right\|^{2}\right]_{+}<\infty$. By reason of (21), we have

$$
\begin{aligned}
& \frac{1}{2} \gamma \eta(1-\lambda \gamma \eta)\left\|(T-I)\left(A y^{k}\right)\right\|^{2} \\
& \leq \varphi_{k}-\varphi_{k+1}+\theta_{k}\left(\varphi_{k}-\varphi_{k-1}\right) \\
&+\theta_{k}\left\|x^{k}-x^{k-1}\right\|^{2}, \\
& \frac{1}{2} \alpha_{k}\left(1-\alpha_{k}\right)\left\|U\left(u\left(y^{k}\right)\right)-u\left(y^{k}\right)\right\|^{2} \\
& \leq \varphi_{k}-\varphi_{k+1}+\theta_{k}\left(\varphi_{k}-\varphi_{k-1}\right) \\
&+\theta_{k}\left\|x^{k}-x^{k-1}\right\|^{2} .
\end{aligned}
$$

Hence,

$$
\begin{gathered}
\sum_{k=1}^{+\infty} \frac{1}{2} \gamma \eta(1-\lambda \gamma \eta)\left\|(T-I)\left(A y^{k}\right)\right\|^{2}<\infty, \\
\sum_{k=1}^{+\infty} \frac{1}{2} \alpha_{k}\left(1-\alpha_{k}\right)\left\|U\left(u\left(y^{k}\right)\right)-u\left(y^{k}\right)\right\|^{2}<\infty .
\end{gathered}
$$

By $\gamma \in(0,1 /(\lambda \eta))$ and the assumption on $\alpha_{k}$, we get

$$
\begin{gathered}
\left\|(T-I)\left(A y^{k}\right)\right\|^{2} \longrightarrow 0, \\
\left\|U\left(u\left(y^{k}\right)\right)-u\left(y^{k}\right)\right\|^{2} \longrightarrow 0 .
\end{gathered}
$$

Denoting by $x^{*}$ a weak-cluster point $\left\{x^{k}\right\}$, let $\left\{x^{k_{\sigma}}\right\}$ be a subsequence of $\left\{x^{k}\right\}$. Obviously,

$$
w-\lim _{\sigma} y^{k_{\sigma}}=w-\lim _{\sigma} x^{k_{\sigma}}=x^{*} .
$$

Then, from (26) and the demiclosedness of $T-I$ at 0 , we obtain

$$
T\left(A x^{*}\right)=A x^{*},
$$

it follows that $A x^{*} \in Q$.

Now, by setting $u_{k}=y^{k}+\gamma \eta A^{*}(T-I)\left(A y^{k}\right)$, it follows that $w-\lim _{\sigma} u^{k_{\sigma}}=x^{*}$. By the demiclosedness of $U-I$ at 0 , from (27), we have

$$
U\left(x^{*}\right)=x^{*} .
$$

Hence, $x^{*} \in C$, and therefore $x^{*} \in \Gamma$.

Since there is no more than one weak-cluster point, the weak convergence of the whole sequence $\left\{x^{k}\right\}$ follows by applying Lemma 5 with $S=\Gamma$.

Remark 7. Since the current value of $\left\|x^{k}-x^{k-1}\right\|$ is known before choosing the parameter $\theta_{k}$, then $\theta_{k}$ is well-defined in Theorem 6. In fact, from the process of proof for Theorem 6, we can get the following assert: the convergence result of Theorem 6 always holds provided that we take $\theta_{k} \in[0, \theta]$, $\theta \in[0,1)$, for all $k \geq 0$, with

$$
\sum_{k=1}^{+\infty} \theta_{k}\left\|x^{k}-x^{k-1}\right\|^{2}<\infty .
$$

To conclude, we have proposed an algorithm for solving the SCFP in the wide class of quasi-nonexpansive operators and proved its convergence in general Hilbert spaces. Next, we will improve the algorithm to assure the strong convergence in infinite Hilbert spaces.

\section{Acknowledgments}

This work was supported by the National Science Foundation of China (under Grant no. 11171221), Shanghai Municipal Committee of Science and Technology (under Grant no. 10550500800), Shanghai Leading Academic Discipline (under Grant no. XTKX 2012), Basic and Frontier Research Program of the Science and Technology Department of Henan Province (under Grant nos. 112300410277 and 082300440150), and China Coal Industry Association Scientific and Technical Guidance to Project (under Grant no. MTKJ-2011-403).

\section{References}

[1] J. W. Chinneck, "The constraint consensus method for finding approximately feasible points in nonlinear programs," INFORMS Journal on Computing, vol. 16, no. 3, pp. 255-265, 2004.

[2] F. Deutsch, "The method of alternating orthogonal projections," in Approximation Theory, Spline Functions and Applications, vol. 356 of NATO Advanced Science Institutes Series C, pp. 105121, Kluwer Academic Publishers, Dordrecht, The Netherlands, 1992.

[3] Y. Censor, "Parallel application of block-iterative methods in medical imaging and radiation therapy," Mathematical Programming, vol. 42, no. 2, pp. 307-325, 1988. 
[4] G. T. Herman, Image Reconstruction from Projections: The Fundamentals of Computerized Tomography, Academic Press, New York, NY, USA, 1980.

[5] Y. Gao, "Determining the viability for a affine nonlinear control system," Journal of Control Theory \& Applications, vol. 26, no. 6, pp. 654-656, 2009 (Chinese).

[6] Y. Censor and T. Elfving, "A multiprojection algorithm using Bregman projections in a product space," Numerical Algorithms, vol. 8, no. 2-4, pp. 221-239, 1994.

[7] Y. Dang and Y. Gao, "The strong convergence of a KM-CQ-like algorithm for a split feasibility problem," Inverse Problems, vol. 27, no. 1, Article ID 015007, 2011.

[8] P.-E. Maingé, "Convergence theorems for inertial KM-type algorithms," Journal of Computational and Applied Mathematics, vol. 219, no. 1, pp. 223-236, 2008.

[9] B. Qu and N. Xiu, "A note on the CQ algorithm for the split feasibility problem," Inverse Problems, vol. 21, no. 5, pp. 1655$1665,2005$.

[10] C. Byrne, "Iterative oblique projection onto convex sets and the split feasibility problem," Inverse Problems, vol. 18, no. 2, pp. 441453, 2002.

[11] Y. Censor and A. Segal, "The split common fixed point problem for directed operators," Journal of Convex Analysis, vol. 16, no. 2, pp. 587-600, 2009.

[12] Y. Dang, Y. Gao, and Y. Han, "A perturbed projection algorithm with inertial technique for split feasibility problem," Journal of Applied Mathematics, vol. 2012, Article ID 207323, 10 pages, 2012.

[13] S. Măruşter and C. Popirlan, "On the Mann-type iteration and the convex feasibility problem," Journal of Computational and Applied Mathematics, vol. 212, no. 2, pp. 390-396, 2008.

[14] H. H. Bauschke and P. L. Combettes, "A weak-to-strong convergence principle for Fejér-monotone methods in Hilbert spaces," Mathematics of Operations Research, vol. 26, no. 2, pp. 248-264, 2001.

[15] A. Moudafi, "A note on the split common fixed-point problem for quasi-nonexpansive operators," Nonlinear Analysis, vol. 74, no. 12, pp. 4083-4087, 2011.

[16] Z. Opial, "Weak convergence of the sequence of successive approximations for nonexpansive mappings," Bulletin of the American Mathematical Society, vol. 73, pp. 591-597, 1967. 


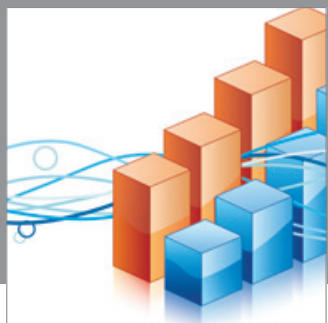

Advances in

Operations Research

mansans

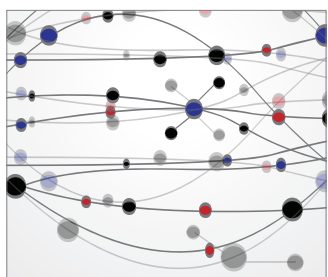

The Scientific World Journal
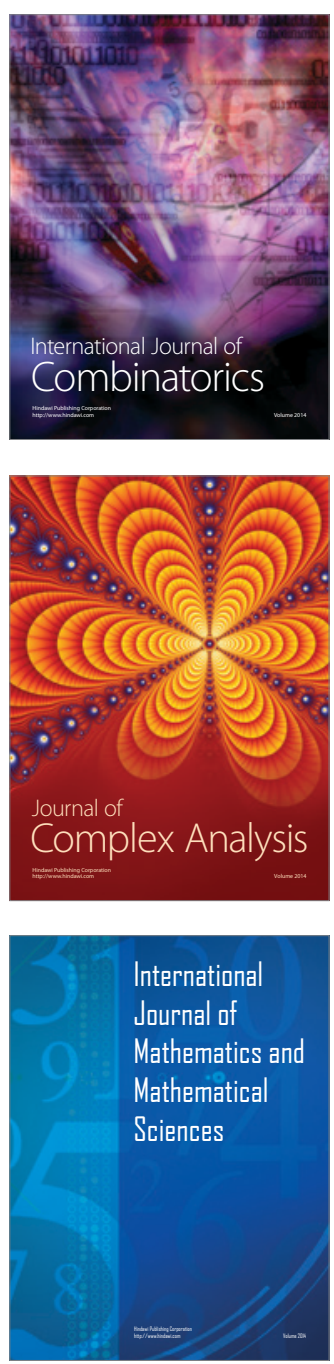
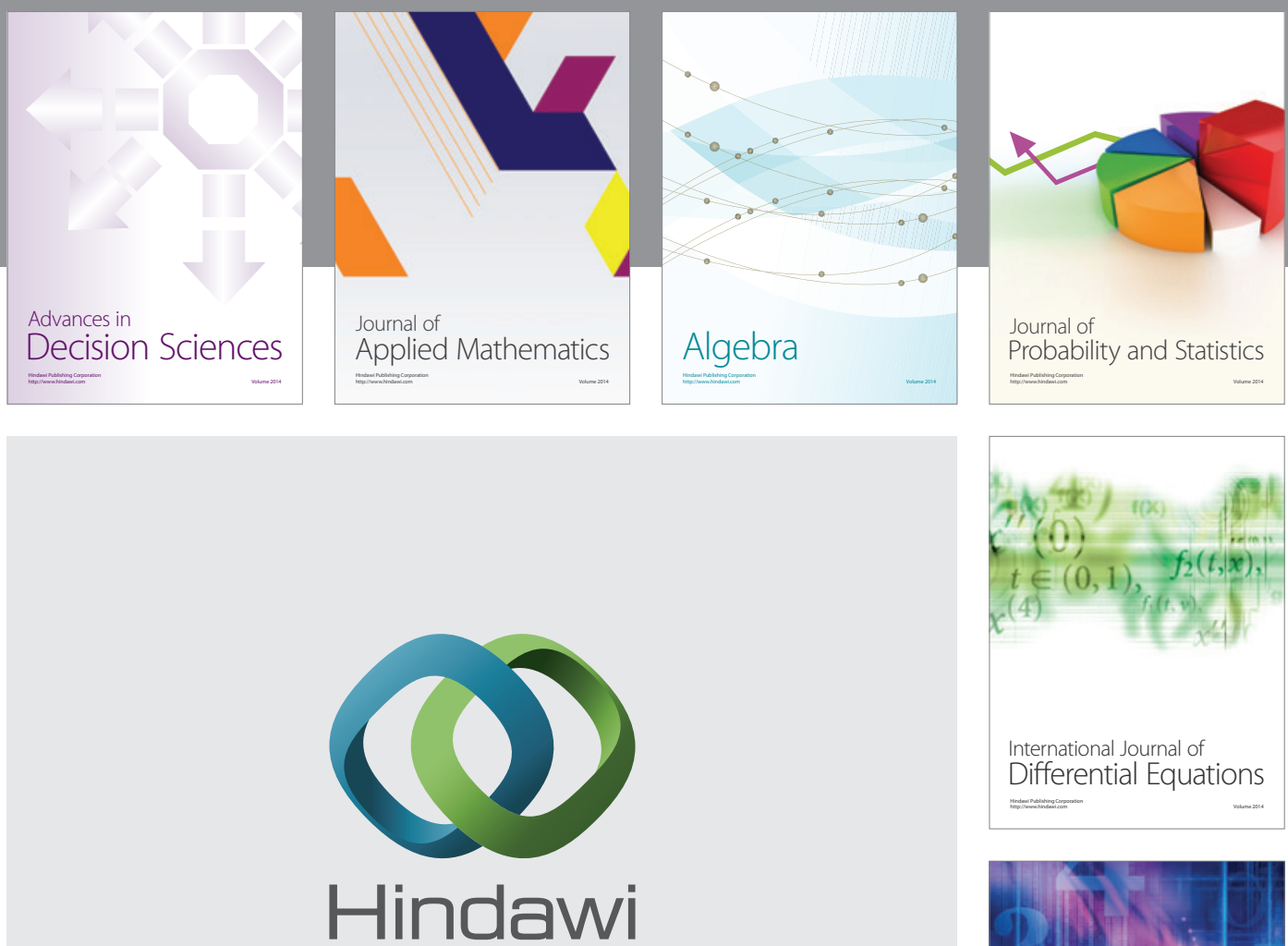

Submit your manuscripts at http://www.hindawi.com
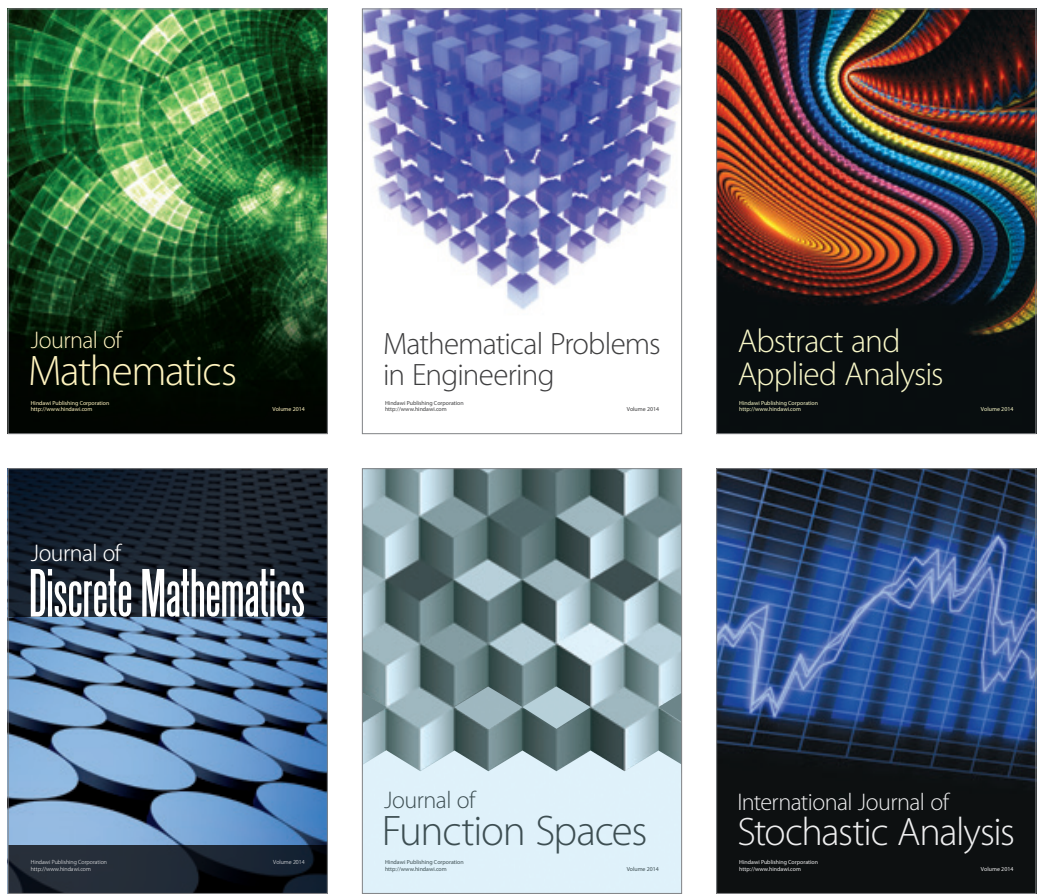

Journal of

Function Spaces

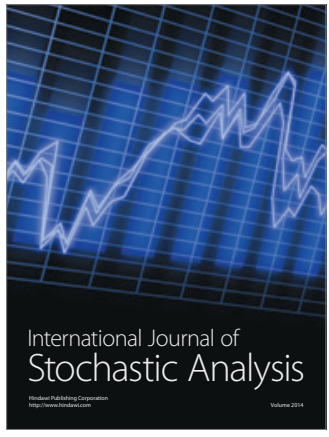

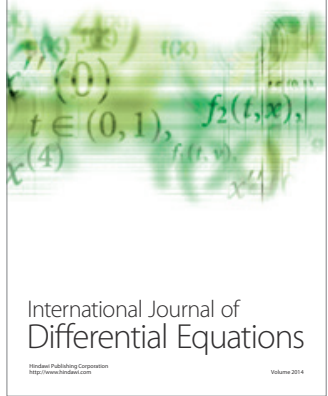
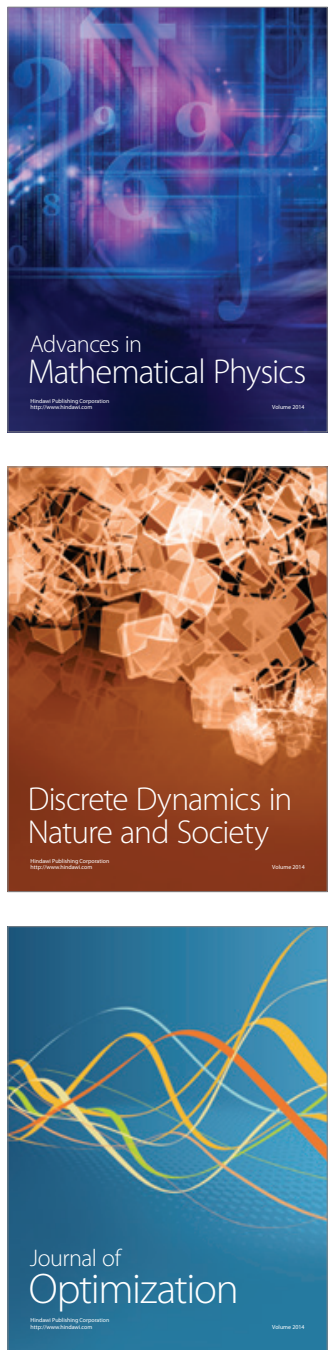